\title{
Extended Bandwidth Optimized and Energy Efficient Dynamic Source Routing Protocol in Mobile Ad-hoc Networks
}

\author{
Bindiya Bhatia ${ }^{1}$, M. K. Soni ${ }^{2}$, Parul Tomar ${ }^{3}$ \\ ${ }^{1}$ Departement of Computer Science \& Engineering, Manav Rachna International University, India \\ ${ }^{2}$ Departement of Electronics \& Communication Engineering, Manav Rachna International University, India \\ ${ }^{3}$ Department of Computer Engineering, YMCA University of Science \& Technology, India
}

\begin{tabular}{l} 
Article Info \\
\hline Article history: \\
Received Jan 12, 2018 \\
Revised Mar 12, 2018 \\
Accepted Mar 26, 2018 \\
\hline
\end{tabular}

Keyword:

Bandwidth optimization

DSR

Energy efficient

Mobile ad-hoc networks

Mobile agents

Routing

\begin{abstract}
With the increase in the evolution of wireless communication, the ad-hoc networks are gaining attention and are significantly becoming the technology solutions to the various problems. Mobile ad-hoc Networks (MANETs) are envisaged to grow as a main component in the today $4 \mathrm{G}$ architecture, and ad hoc networks are projected to be a significant element of the whole future wireless communication. The MANETs are infrastructure less, self-forming and self-organizing network in which there is no control of any centralized entity. The nodes are free to move around the network with dynamic topology. But this self formation, flexibility and scalability create many challenges and design constraints like hidden terminal, limited bandwidth, limited energy of a node, unpredictable change in the topology etc. Bandwidth and energy are the scarce resources of the network. In order to effectively manage the consumption of bandwidth and energy, an algorithm is proposed which is the extension of traditional Dynamic Source Routing (DSR) reactive routing protocol. The extended protocol applies the mobile agents to carry the data. The proposed work is intended to optimize the bandwidth and making the protocol energy efficient.
\end{abstract}

Copyright $@ 2018$ Institute of Advanced Engineering and Science. All rights reserved.

\section{Corresponding Author:}

Bindiya Bhatia,

Departement of Computer Science \& Engineering,

Manav Rachna International Univesity,

Sector 43, Aravali Hills, Surajkund Road, India, pin code-121004.

Email: bindiya.bhatia@gmail.com

\section{INTRODUCTION}

With the increase in the evolution of wireless communication, the ad-hoc network is gaining attention and significantly becoming the technology solution to the various problems. Mobile ad-hoc networks (MANETs) are envisaged to grow as a main component in the $4 \mathrm{G}$ architecture, and projected to be a significant element of the whole future wireless communication. On a broad spectrum, mobile ad-hoc networks are created dynamically through the mobile devices that are linked with each other with wireless links with no pre-existing network infrastructure or any centralized entity. The mobile devices are liberal to roam around the network; thus, the network's topology can be changed swiftly and suddenly. The network can work as a standalone network or can be linked to the Internet. Mobile ad hoc networks don't require any infrastructure viz base station etc for their working. The nodes themselves act as a router and route the data packets through multiple hops. If there is no direct path between the source and the destination, the nodes in the network act as an intermediate node and forward the packets. The mobile device may be a handheld device, tablet, laptop etc. If a node is lying in the transmission range of another node, the two nodes can communicate directly. For interacting with the devices that are outside the transmission range the node take help of its neighbor nodes which forward the packets hop by hop. But this self-formation, flexibility, and scalability create the traditional problems also. The ad-hoc networks have limited bandwidth, energy, and 
computing resources. And the mobility of the devices also creates complex problems of dynamic change in the topology, security, hidden terminal problem etc. The MANET has lower link capacity as compared to the wired links. Although there are various existing routing protocols that are catering to these challenges and providing efficient routing [1]-[3], but these protocols use the significant energy and the bandwidth of the network which are the scarce resources in MANET. Battery power usage of the mobile devices and the link capacity of the network are the major design constraints for forming the ad-hoc networks. It is important that the energy consumption and bandwidth consumption should be effectively managed. So, there is a need for the protocol that is more bandwidth efficient and energy efficient. The various researchers have worked towards the aspects of energy and bandwidth consumption including the study of the behavior of the energy consumption, optimizing the bandwidth, optimizing the energy at the node level, optimizing the energy at the overall network level [4], [5] etc. Even the existing algorithms are extended to provide the energy or bandwidth optimized solutions. But few of the researchers have taken the bandwidth and energy efficiency altogether.

So in order to conserve the energy and make the routing bandwidth optimized, a reactive protocol Dynamic Source Routing (DSR) is optimized and extended to carry the data packets through mobile agents. DSR is a simple and efficient routing protocol for medium size networks. But it doesn't consider the energy of a node and the bandwidth optimization. The main objective of the paper is to make the DSR algorithm energy efficient and bandwidth optimized. And to do so, the algorithm has been extended to use the mobile agents in the route discovery process.

Mobile agents are intelligent program that can move from node to node in a network autonomously and intelligently [6]. They can choose their destination themselves according with the network conditions. The agents can be executed at a node and the execution state of the running program can be saved, and carried to the new node permitting the program to resume where it left the execution on the previous node. The Agents are designed to be as small as possible because a cost is involved to migrate an agent [7], [8]. Mobile agents are very significant implementation technique to disseminate the information in wireless networks [9]-[12].

The main advantages of mobile agent application in MANET are the reduced bandwidth consumption due to the movement of the data processing components to the location of the data and the low energy consumption. From the past few years, research interest has improved in the application of mobile agent in MANET for the various tasks [13]. Mobile agent system provides disconnected operations, code migration and local processing. This facilitates the flexibility and extra capabilities to the ad-hoc networks as compared to ad-hoc networks operations based on traditional client server approach. The approach is highly appreciable when large amount of data is to be collected or disseminated. The mobile agents provide collaborative data processing [14].

The paper is structured into five sections. The next section is describing the literature review of the existing protocols and extensions to optimize the bandwidth and energy. Section 3 is dealing with the explanation of the proposed extended bandwidth and energy efficient DSR routing algorithm. Section 4 gives the simulation result of the proposed modified algorithm and compares its performance with traditional DSR. Section 5 concludes the paper.

\section{LITERATURE REVIEW}

Due to the lack of infrastructure, every node acts as a router to route the packets in MANET. In order to forward the packets, the node requires computing power, bandwidth, energy and other resources. The researchers worked towards making the routing protocols more bandwidth efficient and energy efficient. This section is devoted towards the work of the researchers in the context of bandwidth efficiency and energy efficiency.

Basagni, S., Chlamtac, I., \& Syrotiuk, V. R [15] have extended the DSR protocol and used the geographical positioning of the nodes to make the protocol more bandwidth efficient. In the protocol, a location table is maintained which stores the location of each node as GPS Coordinates. This table provides the information about the network topology and finds the direction of a node from the source. Each node is aware of the position of each node and can also find its neighbor nodes. Thus, routing can be done locally with no requirement of route discovery phase. It minimizes the cost of finding the path and thus minimizes the energy consumption and required bandwidth.

Garcia-Luna-Aceves, J. J., \& Spohn, M [16] have also proposed an algorithm named source tree adaptive routing (STAR) which uses the link state information. The protocol provides bandwidth efficient routing. In the protocol, a source tree is formed by using the information about the links along with favored routes. This tree gives the complete route between a source to a destination. 
Luo, Y., Wang, J., \& Chen, S [17] extended the DSR protocol to make it energy efficient. The protocol finds the route based on the mobility prediction. Garcia, J. E., Kallel, A., Kyamakya, K., Jobmann, K., Cano, J. C., \& Manzoni, P [18] have proposed the energy efficient algorithm which is the extension of DSR. The algorithm tried to not use the nodes in the routing which are having low residual energy. Arango, J., Degermark, M., Efrat, A., \& Pink, S [19] proposed the new flooding algorithm which is bandwidth efficient. It reduces the flooding overheads by minimizing the number of messages needs to reach the destination.

Varaprasad, G., \& Narayanagowda, S. H [20] have modified the DSR algorithm and minimized the energy consumption per packet and maximize the network life time. In the modified algorithm, the three fields are added with the RREQ packet i.e. LSD, bandwidth, and energy model to include the battery power of each node in the route selection.

Srivastava, S., Daniel, A. K., Singh, R., \& Saini, J. P. [21] have proposed the energy efficient position based routing protocol that takes the four parameters into consideration for the route discovery i.e bandwidth, load, energy, and hop count. The proposed algorithm has extended the DSR algorithm by appending some energy constraints. The algorithm chooses the nodes that are not yet much utilized for the routing the packets. It considers the energy of the node, load, hop count and the bandwidth for the selection of the route. If any node found it self not appropriate for forwarding the packet, it will generate the link failure and then the route maintenance phase will be initiated.

Duncan, N., \& Eddie, B. T [22] have also extended the DSR protocol to minimize the routing overhead and thus making it energy efficient. In the protocol, the energy information of the node is also disseminated through the route request packet. Initial the minimum hop count is considered as a metric for route selection but as the node's energy drain out, the node's remaining power is considered as the metric for the selection of the route. The nodes which are having low energy, they don't take part in the routing and don't forward the packet.

With the extensive study of the literature, it can be figured out that the researchers have put attention towards making the routing protocols energy efficient but few of the research work is reported towards making the algorithm bandwidth efficient and energy efficient simultaneously. The next section is proposing an extension of DSR algorithm by applying the mobile agents that are intelligent, autonomous and works on disconnected operations. The proposed algorithm minimizes the utilization of bandwidth as well as making it energy efficient.

\section{PROPOSED MODIFIED DSR PROTOCOL}

The Dynamic Source Routing Protocol (DSR) [23] is an easy and proficient routing protocol intended especially for being utilized in multi-hop mobile ad-hoc networks. Through DSR the network can be entirely self-organized and self-configured. The network does not require any pre- existing infrastructure or any central entity for administration. There are two modules in the protocol. One is "Route Discovery" and another is "Route Maintenance", through which nodes can find and retain the paths from source to destination. The DSR protocol requires more computing power and resources to search for the path. This, in return requires more bandwidth. In order to make the DSR Protocol bandwidth efficient, the protocol is extended $\&$ uses mobile agents in the route discovery process.

The intelligent mobile agents carry the route request packet with them to the next node within the transmission range. The agents will visit the node and search the route cache of the source node to look for the path to the destination node. If the path is there in the route cache, it will come back to the source node with the path else it will move to the next node to search for the path. The route request packet will be contained in the mobile agent. The agent will also have the unique id. The agent doesn't require the bandwidth at the time of searching the visited node's routing table for the destination address. It works on the disconnected process.

The following course of action describes the process of the proposed modified DSR algorithm which is based on the mobile agents:

Step 1: The Sender will check for the local route cache. If there is an unexpired path laying then the path will be appended to the packet header and the data will be routed through this path. All the intermediate nodes will be appended in the node list of the packet header. If this is not the case move to step 2.

Step 2: If the path is not there in the route cache, the sender node will dispatch the mobile agents along with the route request packet. This packet contains the empty node list.

Step 3: The mobile agent will move to the neighbor node with the route request packet. It will look for node's route cache to find the path to the destination node. While this searching and processing, the mobile agent will operate in the disconnected mode and it will not use the bandwidth. Thus the bandwidth and power are optimized. 
Step 4: If the path is found in the route cache of the visited node, the agent will come back with the route reply packet to the source node. The path will be appended to the source routing packet header. Else move to step 5 .

Step 5: If the path is not in the route cache of the visited node then, the visited node will be appended in the node list and the mobile agent will move forward with the packet to the next neighbor node.

Step 6: If the node gets multiple mobile agents with the same route request, the node will accept only the first agent and the rest of the agent will be discarded.

Step 7: The source node will get multiple mobile agents with different paths to the destination. The node will select the path on the basis of minimum hop count.

The packet headers of route request packet and route reply packet are modified as the packets are contained in mobile agents. The format of route request packet is depicted in Figure 1.

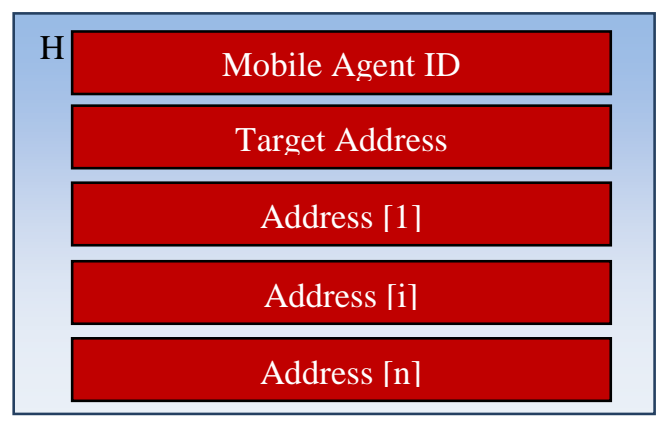

Figure 1. Route request packet

Mobile Agent Id: A unique 16 bit identification ID is allocated to every mobile agent carrying the route request packet. Target Address: It is the 32 bit address of the destination node Address [1]. Address [n] - It is the 32 bit address of the intermediate node through which the mobile agent will visit. Address [1] will be the address of the first node the mobile agent will visit after the source node.

\section{RESULTS AND DISCUSSIONS}

The extended algorithm is simulated with Network Simulator 2(ns2) 2.35 [24]. The simulation area of $1000 \mathrm{~m} * 1000 \mathrm{~m}$ is used to simulate the network. The protocol used is TCP with constant bit rate (CBR) and the mobility model is random way point. Table 1 is detailing all the simulation parameters. With the parameters mentioned in Table 1, the simulation is carried out to simulate the proposed modified DSR algorithm and results are obtained by taking three performance metrics:
a. Packet Deliver Ratio (PDR)
b. Throughput
c. Overall Energy Consumption

Table 1. Simulation Parameter

\begin{tabular}{cc}
\hline Simulation Parameter & Value \\
\hline Simulation time & $1000 \mathrm{~s}$ \\
No. of Nodes & $20,30,40,50$ nodes \\
Simulation Area & $1000 \mathrm{~m}^{*} 1000 \mathrm{~m}$ \\
Mobility model & Random Way point \\
Network Layer Protocol & TCP \\
Traffic Model & CBR \\
Transmission Range & $250 \mathrm{~m}$ \\
Size of the Packet & 512 bytes \\
\hline
\end{tabular}

The simulated results of all the six performance metrics are as follows: a. Packet delivery ratio (PDR)

It is the ratio of a number of packets received at the destination and the number of packets sent from the sender. In the simulation, the PDR is determined by varying the number of nodes and the results in 
Figure 2 show that the PDR of the extended DSR is pretty good as compared to the traditional DSR routing protocol.

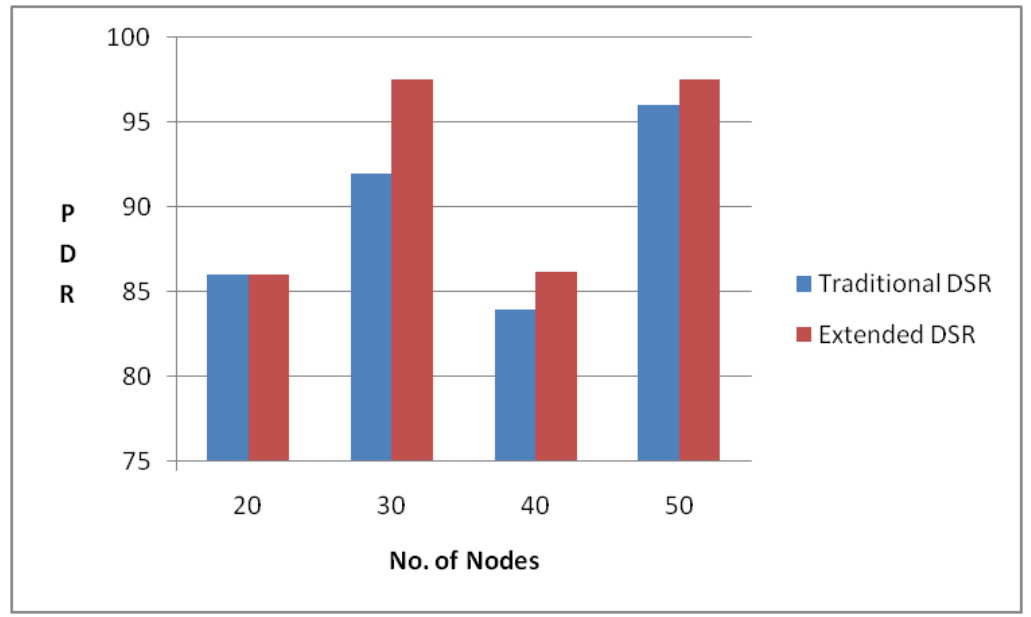

Figure 2. PDR Versus with no. of nodes

b. Throughput

Throughput is the rate at which the packet is transmitted effectively in a total simulation time. The graph in Figure 3 is depicting the high throughput in case of proposed algorithm as compared to traditional DSR as the numbers of nodes are increased.

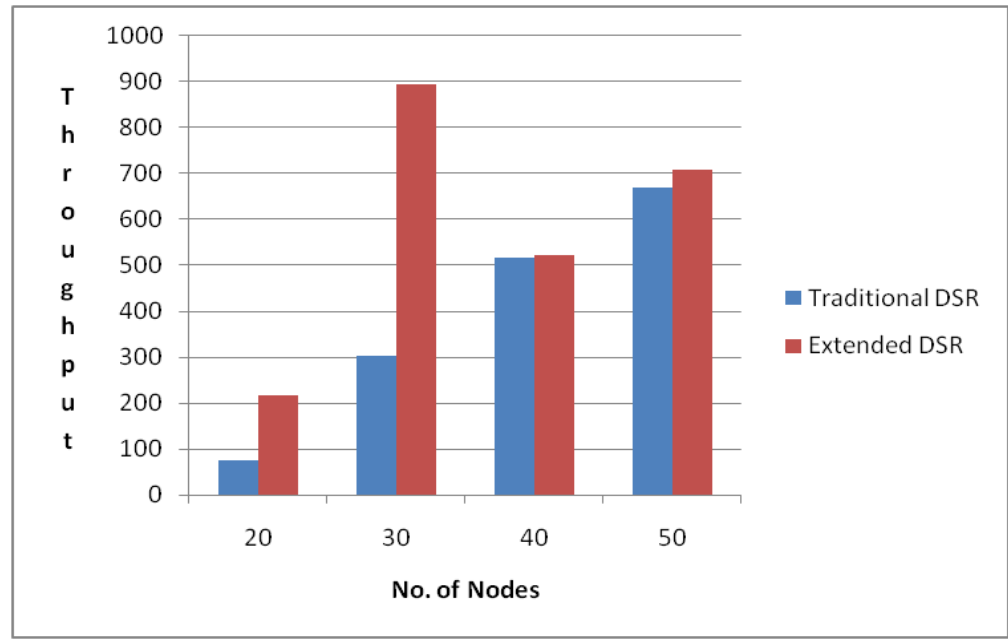

Figure 3. Throughput versus no. of nodes

c. Overall Energy Consumption

It is the total energy utilized during the transmission, reception of the packets through the nodes during a total simulation time. The consumed energy of a node can be calculated as:

Energy Consumed by a node $=\sum$ (Node's energy before the start of the simulation - Node's Residual Energy)

The graph in Figure 4 is showing that the extended DSR is utilizing less energy as compared to DSR. 


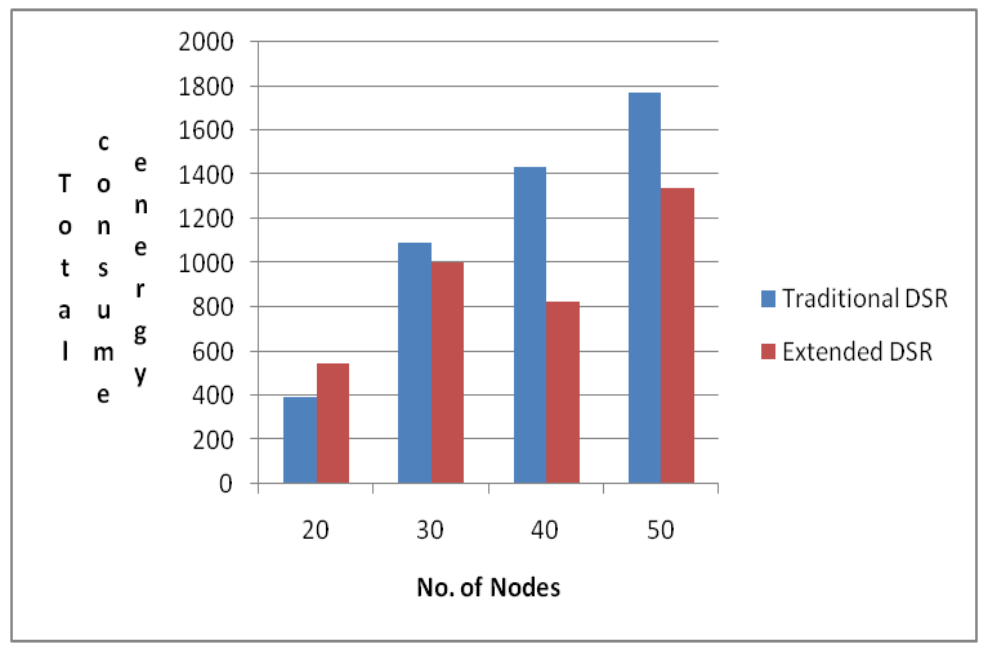

Figure 4. Overall Energy consumption versus no. of nodes

Through these simulation results, it has been found that carrying the packets through mobile agents in DSR algorithm is better as compared to conventional DSR algorithm. It optimizes the energy, bandwidth and hence lowers the delay and increase the packet delivery ratio and throughput.

\section{CONCLUSION}

MANET has lower link capacity as compared to wired networks. And as the mobile devices work on the battery power, the mobile devices in the network have limited energy to operate and forward the data packets. Due to the scarcity of these limited resources, there is a requirement to save these resources. The proposed algorithm based on the application of mobile agents in DSR routing protocol ensures that the bandwidth and the energy can be optimized. The route request packets and the route reply packets are contained in mobile agents and the agents are carrying the packets to the different nodes. The simulation results show that the extended DSR is performing better in the network as compared to traditional DSR in terms of packet delivery ratio, throughput and energy consumption. It is shown in the results that by applying mobile agent, the consumption of total energy and the energy at each node is low. Also the improved PDR and Throughput proved that the bandwidth is optimized.

\section{REFERENCES}

[1] E. Kranakis, et al., "Compass Routing on Geometric Networks," Proceedings of the 11th Canadian Conference on Computational Geometry, 1999.

[2] P. Suma, et al., "Node Disjoint Random and Optimal Path Selection (NDROPS) Algorithm for Security in MANETS," International Journal of Electrical and Computer Engineering (IJECE), vol. 7, 2017.

[3] Z. S. Mahmod, et al., "The Effect of Network's Size on the Performance of the Gateway Discovery and Selection Scheme for MANEMO," Indonesian Journal of Electrical Engineering and Informatics (IJEEI), vol. 5, no. 4, pp. 351-356, 2017.

[4] S. Singh and C. S. Raghavendra, "PAMAS - power aware multi-access protocol with signalling for ad hoc networks," ACM SIGCOMM Computer Communication Review, vol. 28, no. 3, pp. 5-26, 1998.

[5] L. M. Feeney, "Energy efficient communication in ad hoc wireless networks," Mobile Ad Hoc Networking, pp. 301-327, 2004.

[6] I. Satoh, "Mobile agents," in Handbook of Ambient Intelligence and Smart Environments, Springer US, pp. 771-791, 2010.

[7] M. Chen and S. Gonzalez, "Applications and design issues for mobile agents in wireless sensor networks," IEEE Wireless Communications, vol. 14, no. 6, 2007.

[8] D. Chess, et al., "Mobile agents: Are they a good idea? In" Mobile Object Systems: Towards the Programmable Internet," Lecture Notes in Computer Science, vol. 1222, 1997.

[9] N. Migas, et al., "Mobile agents for routing, topology discovery, and automatic network reconfiguration in ad-hoc networks," in Engineering of Computer-Based Systems, 2003. Proceedings. 10th IEEE International Conference and Workshop on the, pp. 200-206, 2003.

[10] A. Patil and S. Sapre, "Intelligent Energy Efficient Routing Protocol based on Biological Agents for MANETS," International Journal of Emerging Technology and Advanced Engineering, vol. 4, no. 7, 2014. 
[11] R. Bindhu, "Mobile agent based routing protocol with security for MANET," International Journal of Applied Engineering Research, vol. 1, no. 1, 2010.

[12] S. A. Putra, et al., "Conceptual Design of Multi-agent System for Suramadu Bridge Structural Health Monitoring System," TELKOMNIKA (Telecommunication Computing Electronics and Control), vol. 13, no. 3, pp. 1079-1088, 2015.

[13] B. Bhatia, et al., "Role of Mobile Agents in the Layered Architecture of Mobile Ad-hoc Networks," International Journal of Computer Network and Information Security, vol. 7, no. 11, pp. 37, 2015.

[14] N. Minar, et al., "Cooperating mobile agents for dynamic network routing," in Software agents for future communication systems, Springer, Berlin, Heidelberg, pp. 287-304, 1999.

[15] S. Basagni, et al., "Dynamic source routing for ad hoc networks using the global positioning system," in Wireless Communications and Networking Conference, 1999. WCNC. 1999 IEEE, vol. 1, pp. 301-305, 1999.

[16] G. L. Aceves, et al., "Source-tree routing in wireless networks," in Network Protocols, 1999. (ICNP'99) Proceedings. Seventh International Conference on, pp. 273-282, 1999.

[17] Y. Luo, et al., "An energy-efficient DSR routing protocol based on mobility prediction," in Proceedings of the 2006 International Symposium on on World of Wireless, Mobile and Multimedia Networks, IEEE Computer Society, pp. 444-446, 2006.

[18] J. E. Garcia, et al., "A novel DSR-based energy-efficient routing algorithm for mobile ad-hoc networks," in Vehicular Technology Conference, 2003. VTC 2003-Fall. 2003 IEEE 58th, vol. 5, pp. 2849-2854, 2003.

[19] J. Arango, et al., "An Efficient Flooding Algorithm for Mobile Ad-hoc Networks," Proceedings of IEEE Workshop on Modeling and Optimization in Mobile, Ad Hoc and Wireless Networks (WiOpt'04). Cambridge, 2004.

[20] G. Varaprasad and S. H. Narayanagowda, "Implementing a new power aware routing algorithm based on existing dynamic source routing protocol for mobile ad hoc networks," IET networks, vol. 3, no. 2, pp. 137-142, 2013.

[21] S. Srivastava, et al., "Energy-efficient position based routing protocol for mobile ad hoc networks," in Radar, Communication and Computing (ICRCC), 2012 International Conference on, pp. 18-23, 2012.

[22] N. Duncan and B. T. Eddie, "An energy-efficient dynamic source routing protocol for mobile ad hoc networks," International Journal of Computing and ICT Research, vol. 2, no. 6, pp. 23-32, 2012.

[23] D. B. Johnson, et al., "DSR: The dynamic source routing protocol for multi-hop wireless ad hoc networks," Ad hoc networking, vol. 5, pp. 139-172, 2001.

[24] NS-2 with Wireless and Mobility Extensions, available via web-site http://www.monarch.cs.cmu.edu

\section{BIOGRAPHIES OF AUTHORS}

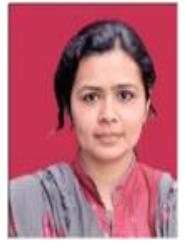

Ms. Bindiya Bhatia is Assistant Professor in Department of Computer Science \& Engineering, Manav Rachna International University, Faridabad. She is pursuing PhD (Computer Science \& Engineering) from Manav Rachna International University. Her major areas of interest are computer networks and agent technology.

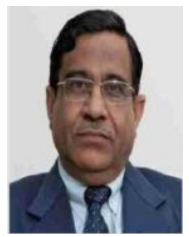

Dr. M. K. Soni is Executive Director \& Dean, Faculty of Engineering \& Technology in Manav Rachna International University, Faridabad. He has done his PhD from National Institute of Technology, Kurukshetra in collaboration with IIT Delhi on Microprocessor and Control Systems. He is having 41 years of experience. His major areas of interest are Networking and Control Systems.

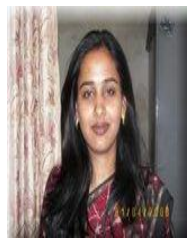

Dr. Parul Tomar received her M.Tech degree in computer Engineering From Maharshi Dayanand University, Rohtak in 2006. Presently, she is working as Assistant Professor in Department of Computer Engineering, YMCA University of Science and Technology, Faridabad, India. She completed her Ph.D. in Computer Science and Applications from Kurukshtra University, Kurukshetra in 2012. Her areas of interest are adhoc networks, Databases, software engineering, programming. 